\title{
Comparison of titanium cable tension band and nickel-titanium patella concentrator for patella fractures
}

\author{
Quan-Ming Zhao ${ }^{A, B, D, F}$, Xiao-Feng Gu ${ }^{A}$, Li Cheng ${ }^{B}$, De-Hong Feng ${ }^{C}$ \\ Department of Orthopedic Surgery, Wuxi People's Hospital, Nanjing Medical University, Wuxi City, China \\ A - research concept and design; $B$ - collection and/or assembly of data; $C$ - data analysis and interpretation; \\ $D$ - writing the article; $E$ - critical revision of the article; $F$ - final approval of article
}

\section{Address for correspondence}

Quan-ming Zhao

E-mail: abc8385@163.com

Funding sources

None declared

Conflict of interest

None declared

\section{Acknowledgements}

This work was supported by the grant from the General Program of Hospital Management Center in Wuxi City, Jiangsu Province (No. YGZXM1536, China.

Received on May 23, 2015

Revised on February 21, 2016

Accepted on April15, 2016

\begin{abstract}
Background. Patellar fractures account for approximately $1 \%$ of all fractures. Due to the patella's importance as regards the extensor mechanism, effort should be made to preserve the patella. Several operative treatment methods have been introduced for patella fractures.
\end{abstract}

Objectives. This study aims to compare the clinical effect of a titanium cable tension band and nickeltitanium (NiTi) patella concentrator (NT-PC) in treating patella fractures.

Material and methods. Thirty-nine patients with patella fractures were enrolled in this retrospective study. All the patients were treated via the open reduction internal fixation procedure using a titanium cable tension band or NT-PC. All the patients were followed up over an average period of 13 months. The main outcome measures were operation time, time of fracture union, postoperative complications, and Böstman knee scores. Statistical analyses were conducted between the 2 groups.

Results. All the patients were operated on successfully. The operation time of the NT-PC treatment group was less than that of the titanium cable tension band treatment group $(p<0.05)$. The mean scores at the final follow-up were 28.2 and 27.6 points in the titanium cable tension band and NT-PC groups, respectively. No significant difference was observed between the excellent and good results $(p>0.05)$.

Conclusions. Both titanium cable tension band and NT-PC showed good efficacy for the treatment of patellar fractures. NT-PC fixation, a new option for the treatment of patella fractures, is a simple and effective fixation method.

Key words: patellar fracture, clinical grading scale of Böstman, patella concentrator

DOI

10.17219/acem/ 62692

\section{Copyright}

Copyright by Author(s)

This is an article distributed under the terms of the

Creative Commons Attribution Non-Commercial License

(http://creativecommons.org/licenses/by-nc-nd/4.0/) 
The patella is the largest sesamoid bone of the human skeleton system. It serves as a fulcrum for the extensor mechanism between the quadriceps tendon and patellar tendon, and provides the mechanical advantage and leverage that increase the force of knee extension. ${ }^{1,2}$ Patellar fractures account for approximately $1 \%$ of all the fractures, and males are affected twice as often as females. ${ }^{3}$ The injury mechanism may be either direct or indirect, and direct trauma is the most common. Additionally, patella fractures can also occur as complications after a total knee replacement surgery or patellar tendon graft transplantation. ${ }^{4,5}$

Because of the patella's importance in the extensor mechanism, efforts are made to restore the patella to its proper position in case of a fracture. The goal of the therapy is the anatomical reduction of both the fracture and articular surface of the patella in addition to the stable fixation of the fracture, facilitating the early rehabilitation of the knee. Surgical treatment becomes necessary when the fracture displacement exceeds $3 \mathrm{~mm}$ or the articular incongruity exceeds $2 \mathrm{~mm}$. Several operative treatment methods have been introduced for comminuted fractures, including lag screws, Kirschner wires, tension band fixation with wires or nonabsorbable sutures with or without screws, and cerclage wiring or a combination of the above. ${ }^{6,7}$ Although metal implants have generally been used for this method of fixation, numerous complications including postoperative pain, K-wire migration, and other complications related to their use are fairly common; therefore, a revision surgery is necessary to remove the implants. ${ }^{8}$ Moreover, far more serious complications such as intra-articular migration of a broken wire have also been reported.

Titanium cable is a new metal implant with superior strength and fatigue resistance; it has replaced wires in upper cervical fusion, arthroplasty, and periprosthetic fractures because of its excellent mechanical properties. Recently, a titanium cable tension band has been developed to treat patellar fracture with satisfactory results. The characteristics of a titanium cable allow it to fix patellar fractures more firmly without any loosening compared to stainless steel wires. The tensile strength and fatigue resistance of a braided titanium cable are 3-6 and 9-48 times more than a traditional stainless steel monofilament wire with a comparable diameter, respectively. When the same force was applied to a stainless steel wire and titanium cable for $24 \mathrm{~h}$, a crack appeared in the stainless steel wire, but not in the titanium cable. Therefore, titanium cable tension band can overcome the disadvantages of common mental implants such as stainless steel wires, K-wires, and screws., ${ }^{9,10}$

A nickel-titanium (NiTi) patella concentrator (NT-PC) has been designed for the initial and continuous compression of patellar fractures. ${ }^{11}$ NT-PC consists of 2 basis patellae claws, 3 apex patella claws, and a conjunctive waist. NT-PC is constructed using nitinol plates of different sizes that have undergone different heat treatments. The final product exhibits a one-way shape memory effect at a phase transformation temperature and reversible deformation. During the implantation, the NT-PC is unfolded in aqua astricta. ${ }^{12}$ The patella concentrator can be easily placed onto a fractured patella. After the operation, the concentrator is warmed and recovers its original shape with a compressive force that fixes the concentrator tightly onto the patella until the fracture is healed. ${ }^{13}$ NT-PC is manufactured according to the anatomical and mechanical characteristics of the patella, which holds the pressure on both the ends of the fracture through the formation of a strong and steady fixation. ${ }^{14}$ According to these characteristics, NT-PC has many advantages including initiative, persistence, multidirectional and centripetal compressive force. NT-PC provides a new method for the treatment of patellar fracture. In this study, we analyzed and compared the clinical effect of titanium cable tension band and NTPC. The aim of this study was to evaluate the efficacy of NT-PC in the treatment of patellar fracture.

\section{Material and methods}

\section{Patients}

Sixteen male and 23 female patients with displaced transverse or comminuted patella fractures (36 closed and 3 open) were screened in this retrospective study. Their ages ranged from 21 to 65 years (mean 45.6 years). All the patients had unilateral patellar fracture caused by direct or indirect violence. Patients were allocated to 2 groups: the titanium cable tension band group (21 patients) and the NT-PC group (18 patients) between August 2011 and June 2014. There was no significant difference in gender, mechanism of the injury or fracture classification between the two groups. All the patients were followed up over an average period of 13 months (range, 6-18 months) after the surgery. Anteroposterior and lateral radiographs were obtained at 3 days, 4 weeks, 8 weeks, and 12 weeks after the surgery, followed by halfyearly follow-ups. The roentgenographic results were independently assessed by radiologists who were not involved in the study. Postoperative complications such as infection, loosening, or breakage of the implants were recorded. The function of the knee was evaluated using Böstman knee scores.

\section{Surgical method}

All surgical procedures were carried out by or under the supervision of experienced senior surgeons. The patients of the titanium cable tension band group were operated on in a supine position. After the preparation and intravenous administration of chemoprophylaxis, a thigh tourniquet was inflated. The fracture was ex- 
posed through midline longitudinal incision. When the retinaculum was disrupted, the reduction maneuver involved passing a temporary suture around the patella to stitch the fragments together. Then, the articular surface was palpated, and the reduction was adjusted accordingly through arthrotomy. The final reduction was checked using an image intensifier. A cable was passed circumferentially around the patella forming an "O," and the other titanium cable strengthened the fixation with an " 8 " form in prepatellar. The cables were tensioned to $30 \mathrm{lbs}$. The articular surface was checked again using palpation and image intensifier, and the knee was flexed to $90^{\circ}$ to confirm that the fragments do not separate more than $1 \mathrm{~mm}$ before crimps were applied and the excess cables were cut. The soft-tissue envelope on the patella was then repaired with a suture, and the wound was closed in layers.

To the NT-PC group, similar to the titanium cable tension band group, a longitudinal midline skin incision was made over the patella to reach the fracture site, and the hematoma was drained. Care was taken to avoid damage to the vessels of the genicular arteries, followed by an enbloc preparation of the fasciocutaneous layers under the bursa prepatellaris. Under direct vision, an anatomical reconstruction was performed using one or several bone reduction forceps; the smaller fracture fragments were reduced by the suture of the fasciocutaneous layers with an absorbable surgical suture. Thus, a complex fracture pattern was simplified. An appropriately sized NT-PC was selected and soaked in $0^{\circ} \mathrm{C}-4^{\circ} \mathrm{C}$ ice salt water. Small longitudinal incisions were made at the upper and lower ligaments of the patella. The open branch and waist of the NT-PC was split with the opening tongs prior to insertion into the upper and lower borders of the patella. Heat $\left(45^{\circ} \mathrm{C}\right)$ was applied to the NT-PC through a saline gauze to restore the original shape and close around the patella tightly. The anatomical reduction of the articular surface was checked by X-ray and digital palpation of the patellofemoral joint inside the knee. Finally, the quality of the reduction was rechecked, and the knee was gently flexed to assess the stability of the fixation. The soft-tissue envelope on the patella was then repaired by suturing, and the wound was closed in layers.

\section{Postoperative management}

Both active and passive knee motion exercises began 1 day after the surgery. On postoperative day 1 , isometric exercises involving the quadriceps were initiated. On postoperative day 2, partial or full weight-bearing exercises were started under the protection of a hinge brace. On postoperative day 3, walking exercises were performed using crutches. One month after the surgery, normal daily activities for the participants were permitted. Three months after the surgery, modest sports activities were permitted. X-ray examination was performed through outpatient recheck to observe the fracture healing.

\section{Results}

The mean operation times were 68 min (range, 52$-76 \mathrm{~min}$ ) in the titanium cable tension band group and $56 \mathrm{~min}$ (range, 50-68 $\mathrm{min}$ ) in the NT-PC group. A statistical difference was observed between the 2 groups $(\mathrm{p}<0.05)$. The mean times between the operation and union were 60 days (range, 53-72 day) in the titanium cable tension band group and 63 days (range, 51-78 day) in the NT-PC group. No statistical difference was observed in bone union between the 2 groups ( $p>0.05)$. No postoperative complications such as infection, dislocation, or breakage of the implants were observed through the final follow-up examination in the 2 groups (Fig. 1 and 2).

According to the method of Böstman et al., satisfactory results were obtained for the recovery of the knee function. The mean score at the final follow-up was 28.2 points (range $25-30$ points) in the titanium cable tension band group. Nineteen patients $(90.5 \%)$ had an excellent result, 2 patients (9.5\%) had a good result, and no patients had an unsatisfactory result. The mean score at the final follow-up was 27.6 points (range $25-30$ points) in the NT-PC group. ${ }^{15}$ Fifteen patients $(83.3 \%)$ had an excellent result, 3 patients $(16.7 \%)$ had a good result, and no patients had an unsatisfactory result. No significant difference was observed in the excellent and good results ( $\mathrm{p}>0.05)$ (Table 1).

\section{Discussion}

In this study, 2 different methods used for the internal fixation of patella fractures were compared. The clinical effects of 2 established methods, titanium cable tension band and NT-PC fixation, were analyzed. No significant difference in the functional results was observed between the 2 methods. For the titanium cable tension band fixation, the functional results were excellent (81\%) and good (19\%). For the NT-PC fixation, the functional results were excellent (81\%) and good (19\%). This indicates a similar clinical effect of titanium cable tension band and NT-PC in treating patella fractures.

The tension band technique is most often used for patella fractures with satisfactory results. ${ }^{16}$ However, conventional metal implants such as stainless steel wires, K-wires, and screws cause numerous complications including postoperative pain, K-wire migration, and other complications. A revision surgery is necessary to remove the implants. Besides, although a stainless steel wire has a certain elasticity, it does not close the patella surface. Moreover, a stainless steel wire is tightened by hand, and it is not easy to control the degree of tightness. A stainless steel wire may cut the patella surface if it is too tight; thus it is not appropriate for patients with osteoporosis. On the other hand, a stainless steel wire does not act as a tension band if it is too loose. 
Table 1. Details of the clinical grading scale of Böstman et al.

\begin{tabular}{|c|c|}
\hline $\begin{array}{l}\text { Range of movement (ROM) } \\
\text { full extension and the ROM }>120^{\circ} \text { or } \\
\text { within } 10^{\circ} \text { of the normal side } \\
\text { full extension, movement } 90^{\circ} \text { to } 120^{\circ}\end{array}$ & $\begin{array}{l}6 \\
3\end{array}$ \\
\hline $\begin{array}{l}\text { Pain } \\
\text { none or minimal on exertion } \\
\text { moderate on exertion } \\
\text { in daily activity }\end{array}$ & $\begin{array}{l}6 \\
3 \\
0\end{array}$ \\
\hline $\begin{array}{l}\text { Work } \\
\text { original job } \\
\text { different job } \\
\text { cannot work }\end{array}$ & $\begin{array}{l}4 \\
2 \\
0\end{array}$ \\
\hline $\begin{array}{l}\text { Atrophy, difference of circumference of } \\
\text { thigh } 10 \mathrm{~cm} \text { proximal to the patella } \\
<12 \mathrm{~mm} \\
2-25 \mathrm{~mm} \\
>25 \mathrm{~mm}\end{array}$ & $\begin{array}{l}4 \\
2 \\
0\end{array}$ \\
\hline $\begin{array}{l}\text { Assistance in working } \\
\text { none } \\
\text { can part of the time } \\
\text { can all the time }\end{array}$ & $\begin{array}{l}4 \\
2 \\
0\end{array}$ \\
\hline $\begin{array}{l}\text { Effusion } \\
\text { none } \\
\text { reported to be present } \\
\text { present }\end{array}$ & $\begin{array}{l}2 \\
1 \\
0\end{array}$ \\
\hline $\begin{array}{l}\text { Giving way } \\
\text { none } \\
\text { sometimes } \\
\text { in daily life }\end{array}$ & $\begin{array}{l}2 \\
1 \\
0\end{array}$ \\
\hline $\begin{array}{l}\text { Stair-climbing } \\
\text { normal } \\
\text { disturbing } \\
\text { disabling }\end{array}$ & $\begin{array}{l}2 \\
1 \\
0\end{array}$ \\
\hline $\begin{array}{l}\text { Total score } \\
\text { excellent } \\
\text { good } \\
\text { unsatisfactory }\end{array}$ & $\begin{aligned} & 30 \text { to } 28 \\
& 27 \text { to } 20 \\
< & 20\end{aligned}$ \\
\hline
\end{tabular}

Titanium cable tension band is the ideal method of treatment for a patella fracture, and it fully utilizes the advantages of a K-wire tension band. More importantly, a titanium cable has many advantages. First, it has a small size, good biocompatibility, nontoxic side effects; the histocompatibility of titanium is good enough to minimize the scar tissue caused by internal implants. Second, a titanium cable is produced from titanium metal, and its tensile strength, fatigue resistance, and wear resistance are better than that of a stainless steel wire. Moreover, the strength of a titanium cable is similar to that of a cortical bone. When tightened, a titanium cable can be closely attached to the bone and does not break. Additionally, special equipment is used to tighten a titanium cable tension band. An operator can control the degree of tightness to the maximum extent; this is especially important for osteoporotic patients. ${ }^{17,18}$ Because of these advantages of a titanium cable, patellar fractures can be fixed more firmly without causing loosening, thus facilitating the early functional exercise of the knee joint.
Fig. 1. X-ray images of NT-PC treatment of patellar fracture. A 56-year-old male patient injured in a traffic accident
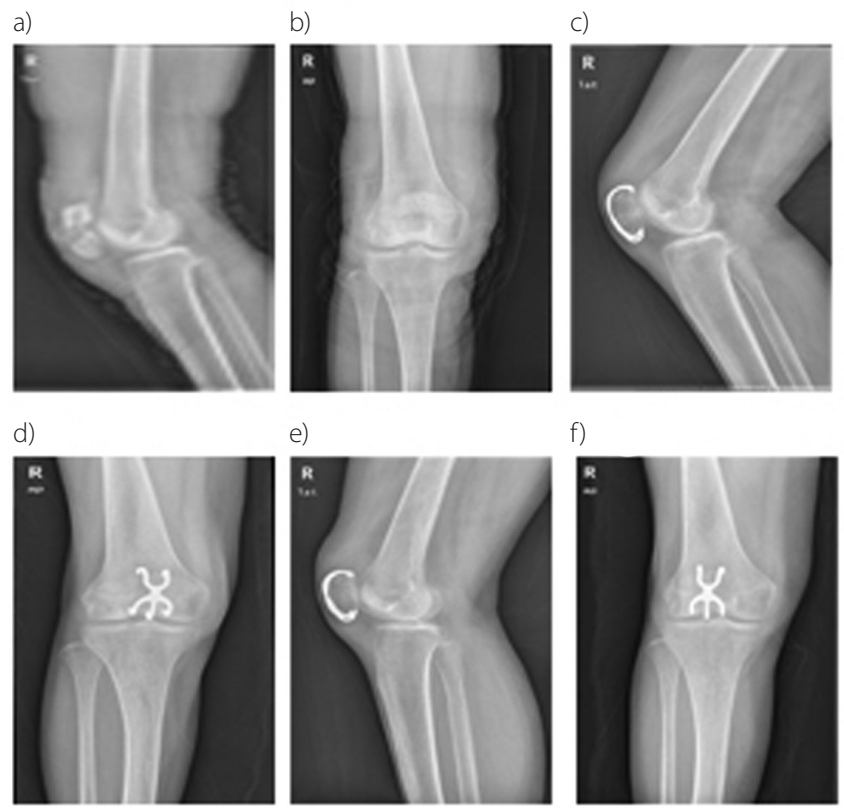

a) and b): presurgery; c) and d): four-weeks postsurgery; e) and f): twelve-weeks postsurgery.

Fig. 2. X-ray images of titanium cable tension band treatment of patellar fracture. A 68-year-old male patient injured by falling down
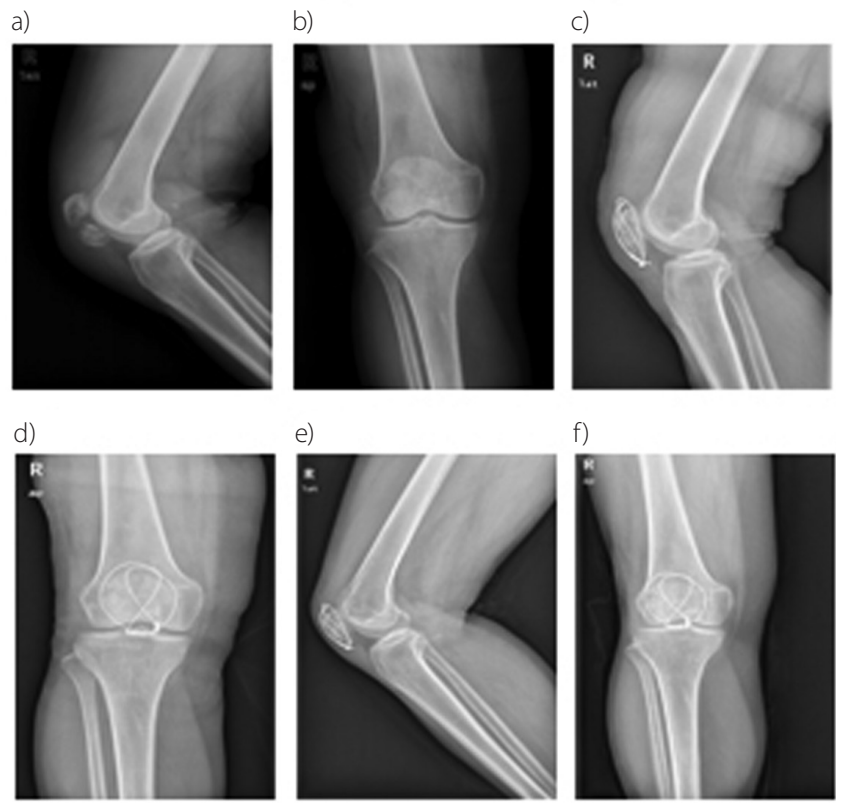

a) and b): presurgery; c) and d): four-weeks postsurgery; e) and f): twelve-weeks postsurgery.

Similarly, NT-PC fixation provides an efficient method for the treatment of patellar fractures, especially for the comminuted fracture of patella. The shape is similar to a starfish with a total of 5 claws radiated, and the outline approaches the physiological radian of the patella. Because NT-PC has mechanical and anatomical characteristics, it has a dual function of reduction and fixation. Additionally, NT-PC provides a multidirectional, concentric, and continuous force to encircle the heart to press 
the broken ends of the fracture automatically, thus providing sufficient fracture stability. Further, the anatomy of the articular surface of the patellofemoral can further restore the knee joint activities according to the principles of the tension band of patella claws.

Particularly, NT-PC has a shape memory function, producing memory and resilience when the temperature changes from low to high. In the process, NT-PC applies force from different directions to the center of the patella, thus firmly fixing the fracture and resisting the stress of 4 quadriceps. Moreover, it is unnecessary to drill and wedge other auxiliary internal fixation during the reduction. The operation is simple, and the operation time is short. The operator only needs to arrange the claws precontoured in advance around the patellar fracture, fixing it firmly; therefore, no further damage is done to the blood supply of the patella, contributing to fracture healing.

However, nickel ions released from alloys are potent allergens or haptens that can trigger skin inflammation. The clinical symptoms of nickel allergy include rashes, swelling, pain and etc. They penetrate the skin and activate epithelial cells that produce various cytokines or chemokines. ${ }^{19}$ So NT-PC has the danger of nickel allergy potentially.

This is the first study to compare the curative effects of 2 types of internal fixation for treating patella fracture, not reported earlier. This study is limited by a small number of patients. Further studies should be conducted in the future.

In conclusion, the 2 different fixation methods for the treatment of patellar fractures, titanium cable tension band and NT-PC, showed good results. NT-PC fixation, a new option for the treatment of patellar fractures, is a reliable method.

\section{Reference}

1. Kakazu R, Archdeacon MT. Surgical management of patellar fractures. Orthop Clin North Am. 2016;47(1):77-83.

2. Wild $\mathrm{M}$, Windolf J, Flohé $\mathrm{S}$. Fractures of the patella. Unfallchirurg. 2010;113:401-412.
3. Böstman O, Kiviluoto O, Santavirta S, Nirhamo J, Wilppula E. Fractures of the patella treated by operation. Arch Orthop Trauma Surg. 1983;102:78-81.

4. Radło W, Lorkowski J. Congenital dislocation of the patella in children and adolescents. Chir Narzadow Ruchu Ortop Pol. 1997;62(4):331-335.

5. Witoński D, Keska R, Bira M. Reconstruction of the medial patellofemoral ligament with patellar tendon autograft - preliminary report. Chir Narzadow Ruchu Ortop Pol. 2010;75(1):42-46.

6. Matsuo T, Watari T, Naito K, Mogami A, Kaneko K, Obayashi O. Percutaneous cerclage wiring for the surgical treatment of displaced patella fractures. Strategies Trauma Limb Reconstr. 2014;9(1):19-23.

7. Della Rocca GJ. Displaced patella fractures. J Knee Surg. 2013;26: 293-299.

8. LeBrun CT, Langford JR, Sagi HC. Functional outcomes after operatively treated patella fractures. J Orthop Trauma. 2012;26:422-426.

9. Maniar RN, Nayak RM, Vatchha S, Singhi T. Periprosthetic patellar fracture fixation using suture anchors. Orthopedics. 2013;36(11):e1470-1473.

10. Sayeed SA, Naziri Q, Patel YD, Boylan MR, Issa K, Mont MA. Patellar fractures following total knee arthroplasty: A review. J Long Term Eff Med Implants. 2013;23(4):331-336.

11. Tang Y, Zhang YT, Fu QG, Zhang CC, Zhang X, Wang PF. Application of $\mathrm{Ni}$-Ti patellar concentrator combined with Herbert screw and wirerope for the treatment of comminuted patellar inferior pole fractures. Zhongguo Gu Shang. 2013;26:457-459.

12. Zhang $Y$, Wang $P$, Zhang $C$, Fu Q, Zhang $X$. Management of inferior polar comminuted fractures of patella and patellar tendon injury. Zhongguo Xiu Fu Chong Jian Wai Ke Za Zhi. 2011;25:976-979.

13. Wada K, Liu Y. Thermomechanical training and the shape recovery characteristics of NiTi alloys. Mater Sci Eng A. 2008;481:166-169.

14. Hao W, Zhou L, Sun Y, Shi P, Liu H, Wang X. Treatment of patella fracture by claw-like shape memory alloy. Arch Orthop Trauma Surg. 2015;135(7):943-951.

15. Böstman O, Kiviluoto O, Nirhamo J. Comminuted displaced fractures of the patella. Injury. 1981;13:196-202.

16. Yu XL, Xu C, Li SD, Zhan JD, Xu ZQ. Progress on treatment of transverse patella fractures with tension band fixation. Zhongguo Gu Shang. 2015;28(11):1069-1074.

17. Rathi A, Swamy MK, Prasantha I, Consul A, Bansal A, Bahl V. Percutaneous tension band wiring for patellar fractures. J Orthop Surg. 2012;20:166-169.

18. Dickens AJ, Salas C, Rise L, et al. Titanium mesh as a low-profile alternative for tension-band augmentation in patella fracture fixation: A biomechanical study. Injury. 2015;46(6):1001-1006.

19. Saito M, Arakaki R, Yamada A, Tsunematsu T, Kudo Y, Ishimaru N. Molecular mechanisms of nickel allergy. Int J Mol Sci. 2016;17(2):202. 